\title{
Direct Drug Delivery to Kidney via the Renal Artery
}

Ulrika Dahlqvist ${ }^{1}$, Tajana Tesan Tomic ${ }^{2}$, Magnus Söderberg ${ }^{3}$, Jane Stubbe ${ }^{4}$, Camilla Enggaard ${ }^{4}$, Anette Ericsson ${ }^{5}$, AlexXianghua Zhou ${ }^{1}$, Anna Björnson Granqvist ${ }^{1}$, Lena William-Olsson ${ }^{1}$

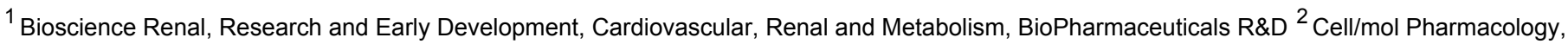

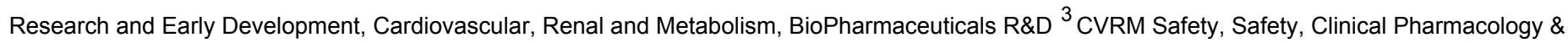

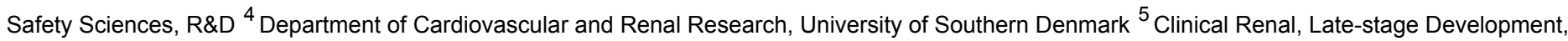
Cardiovascular, Renal and Metabolism, BioPharmaceuticals R\&D

\section{Corresponding Author}

Ulrika Dahlqvist

ulrika.dahlqvist@astrazeneca.com

\section{Citation}

Dahlqvist, U., Tomic, T.T.,

Söderberg, M., Stubbe, J., Enggaard, C., Ericsson, A., Zhou, A.X., Björnson

Granqvist, A., William-Olsson, L. Direct Drug Delivery to Kidney via the Renal Artery. J. Vis. Exp. (170), e61932, doi:10.3791/61932 (2021).

\section{Date Published}

April 17, 2021

\section{DOI}

$10.3791 / 61932$

URL

jove.com/video/61932

\section{Abstract}

There is a need for directed injections to enable increased and specific renal exposure for efficient evaluation of drug targets in the renal research field. Accumulation of drugs in certain organs may give rise to adverse and unwanted effects, depending on the nature of injectate. To minimize spillover and/or accumulation in other tissues, the herein described method directs the formulation into the renal artery bloodstream by inserting a catheter in the infra renal aorta, just below where it branches into the renal artery, resulting in the kidney as first reached organ and distributing of formulation throughout the kidney.

This manuscript provides a detailed description of the method, as well as its challenges and difficulties. It guides the experimenter to become skillful with this type of microsurgery that requires accuracy under sterile conditions. Speed is crucial for minimizing the ischemia and practicing the procedure will increase the chance of successful injections without adverse effects. By modulating the time between injection and reperfusion as well as the injected volume, the risk of spillover to other organs is mitigated.

Note that this technique is suitable for single dosing strategies.

\section{Introduction}

In the preclinical research field within the pharmaceutical industry, model- and method- development is part of the daily work. There is an increasing interest for the ability to direct drugs to specific organs, or even separate compartments of an organ, without major spillover/entrapment, via bloodstream, into other tissues. This to enable increased and specific exposure for efficient evaluation of drug targets in models of different disease areas $1,2,3,4$.

A common way of delivering substances is by systemic routes (e.g., via the tail vein) since it is less invasive than the method described in this manuscript. However, systemic 
administration increases the risk of increased metabolism or accumulation of the compound in other tissues than the intended target organ, when passing through filtering organs such as the lung, the liver and the spleen ${ }^{2,3,5}$. Aside from not reaching the destined tissue, this could potentially give rise to adverse and/or unwanted effects, depending on the nature of injectate. Only very small molecules pass the capillaries of filtering organs and therefore targeted delivery is especially important if working with larger molecules ${ }^{6}$.

To minimize spillover and/or accumulation of injected formulation, in other tissues, the herein described method directs the formulation into the renal artery bloodstream through a catheter inserted in the abdominal aorta just below where it branches into the renal artery, resulting in the kidney as first reached organ. Another advantage with this guided administration is that a lower dose/volume can be used in order to reach the same level of exposure as achieved via systemic administration ${ }^{3}$.

Other routes of administration have been explored, for example, injections via a catheter directly into the renal artery. In our hands, this was found to present a higher risk of failure to restore the circulation to the kidney. The very small diameter of the renal artery (approximately $0.35-0.55 \mathrm{~mm}$ in diameter) makes the incision relatively large and pose a risk of obstruction and/or embolism when closing the entry hole. According to our experience ischemic-related damage to the kidney often occurred when using this method, and we therefore developed this new way of successfully target the kidney by injecting substances through incision of the larger aorta to target the kidney.

There are similar techniques being developed in rats that also manifests the challenges and risk of stenosis/thrombosis working with injections directly into the renal artery ${ }^{5}$. This supports our findings since the vessels in mice are even smaller.

This manuscript and video describe, in detail, how injections can be directed into the renal artery in mice through a catheter inserted in the infrarenal aorta, as well as guidance in how to overcome common difficulties in the procedure, to work in the safest way possible and thereby increase reproducibility.

\section{Protocol}

Experimental procedures were approved by the Regional Laboratory Animal Ethics Committee of Gothenburg, Sweden.

\section{Preoperative care}

1. Use sterile techniques to avoid infections.

1. Under anesthesia (e.g., isoflurane) and working in the most aseptic way possible, shave the surgery area and remove the fur with a hair removal cream. Leave the cream for around 1 minute (maximum of 2 minutes to avoid skin-burns). Wash carefully with water. This first step can preferably be done 1-2 days before surgery to avoid extra cooling of the animal right before surgery.

2. Apply ophthalmic lubricant on the eyes to prevent dryness.

3. To avoid infections, wash the surgical area with antiseptic skin cleanser and wipe the area with disinfectant (chlorhexidine) right before surgery.

2. Analgesia

1. Pre-dose the animal with analgesics if it is not a terminal procedure: Buprenorphine (e.g., temgesic) $0.05-0.1 \mathrm{mg} / \mathrm{kg} \mathrm{s.c.)}$ 
3. Anticoagulants

1. Use anticoagulants to avoid blood clots: $10 \mathrm{IU}$ of heparin through the tail vein right before surgery (maximum $5 \mathrm{~mL} / \mathrm{kg}$ ).

\section{Surgical procedure}

1. Autoclave the instruments and use sterile catheters. Have the surgent wash up with soap and hibiscrub and wear a facemask, a hairnet and a sterile surgical gown and gloves. Have a second person handle the animals and all "dirty" tasks.

2. For the anesthesia, use isoflurane. Induce anesthesia by placing the mouse in an anesthesia-box with $5 \%$ isoflurane. After the induction, check the depth of anesthesia by checking reflexes and breathing.

1. During surgery, place a facemask on the mouse to give a maintenance dose of $2 \%$ isoflurane.

3. Wash the animal with hibiscrub and chlorhexidine before putting on the surgical table.

4. After anesthetizing, place the mouse on a heated surgical pad in a supine position and fix the upper and lower limbs to the pad using low-tack adhesive tape. Ensure that the upper extremities are maintained in a normal position to prevent lung compression. If possible, use a non-electric heat source.

NOTE: We use inhalation anesthesia (e.g., isoflurane) for easier adjustments during surgery.

5. Drape the mouse with plastic wrap (e.g., Press'n Seal) and perform the work with a surgical microscope.

6. Protect the tissues from drying by constantly adding saline into the surgery area. This also helps keeping the tissue elastic and minimizes risk of damage while performing the surgery. The saline should be around 37 ${ }^{\circ} \mathrm{C}$ to avoid cooling of the animal.

7. After opening the abdominal cavity with a midline incision with forceps and scissors, use a moist, folded, compress to push all other organs aside to get a good view over the surgery area.

8. Use retractors to have a good overview of the surgery area (5 $\mathrm{mm}$ blunt hook).

9. Localize the aorta and the left kidney.

10. Using two microsurgery forceps, gently clean the aorta, both cranially (see 2.10.1) and caudally (see 2.10.2) fromthe kidney, from surrounding tissues and place a ligature underneath it (6-0 Silk). Be careful not to pinch any structures. Work your way in by gently stretching and pulling the tissues, getting as close to the renal artery as possible. Around the vessels there are several nerves not easily discovered. Keep this in mind since damaged nerves can cause paralysis or other issues post operatively.

1. Cranially (above) the kidney, use this ligature to lift the aorta to momentarily occlude the blood flow while performing the injection. In this position, right above where the renal artery, the aorta branches in the opposite direction (to the intestines, see Figure 1). In order to avoid pushing the injection this way, put the ligature beneath this branch. Leave the ligature without any tension until starting the injection procedure.

2. Caudally (below) the kidney, place the incision close to where the aorta branches to the kidney/renal artery to avoid other potential branches. Caudally from the kidney the aorta is very close to the 
abdominal vein. Be very careful not to damage the vein.

11. Injection

1. When all ligatures are in place; first stretch the lower and secondly the upper ligature to occlude the blood stream to direct the injected fluid to the kidney.

2. Using an acupuncture needle $(\varnothing 0.25 \mathrm{~mm})$, puncture and guide the (rounded) tip of the catheter (32GA) into the aorta and secure it with a single knot on the ligature so that it will not move or leak during injection. (Figure 1). Using an acupuncture needle instead of a scissor enable the making of a minimal entry hole in the vessel.

3. Inject $50 \mu \mathrm{L}$ of fluids. The kidney should turn pale, indicating that the injection has perfused the kidney and ended up in the destined place.

4. At this point there are two different scenarios possible.

1. Give some circulation back to the kidney by carefully loosen the tension in the upper ligature before closing the entry hole. This way ischemia is ameliorated but be aware that the injected fluid can potentially be cleared rapidly via the renal vein and thus not allowing enough dwell time.

2. Or proceed with surgery without giving blood flow back.

5. After tightening the upper ligature again, withdraw the catheter, suture the aorta and give back full circulation.

1. Remove the ligature holding the catheter.

2. Withdraw the catheter.
3. Suture the entry hole with a single stitch (ethilon $11-0$, rounded needle).

4. First loosen the lower ligature and then very slowly loosen the upper one. If there is leakage tighten the ligature again, wait for a few seconds and try again. Keep the total ischemia time to a maximum of 5-10 minutes to avoid ischemic damage.

5. Test the solidity by pushing the area and look for pulsations and see to that the kidney returns to "normal coloring" to reinsure that recirculation is established.

6. Cut the threads and remove the ligatures underneath aorta.

7. Remove the compress and push the intestines back in place. Give back some fluids, by adding saline into the abdominal cavity and suture the peritonea (Vicryl 6-0) before closing the skin with metal clips.

8. Before waking up the animals are given $0.7-1$ $\mathrm{mL}$ of Rehydrex subcutaneously.

NOTE: The whole procedure takes around 30-45 minutes for a well-trained surgent.

\section{Postoperative care}

1. Allow animals to wake up in a warm environment by placing a heating pad (around $38^{\circ} \mathrm{C}$ ) underneath part of the cage for 2-3 days post operatively.

2. Continue providing analgesia (Buprenorphine, e.g., temgesic $0.05-0.1 \mathrm{mg} / \mathrm{kg}$ s.c.) $2-3$ times/day for 3 days post operatively. 
3. Give $10 \mathrm{~mL} / \mathrm{kg}$ of Rehydrex s.c. occasionally if animals show signs of dehydration.

\section{Representative Results}

The film is a mix of videos from different surgeries to present the ones with the best video quality. Some sequences have been taken from practice sessions where the mouse is not supposed to wake up. Therefore, the mouse is not always properly draped. When an animal is supposed to wake up, we always work in an aseptic way.

Histological analyses revealed that performing the injections directly into the renal artery gave rise to kidney injury, likely due to insufficient recirculation of blood flow with irreversible ischemia as a result (Figure 2). Injection through the abdominal aorta, on the other hand, did not cause any ischemic injury (Figure 3).

Evans blue dye was used to visualize that perfusion via the aorta will result in the kidney being the first reached organ (Figure 4). No other organs turned blue until after the passage out through the renal vein.

We have also showed that using this method we can increase engraftment of injected formulations to the perfused kidney using mesenchymal stem cells (Figure 5). 

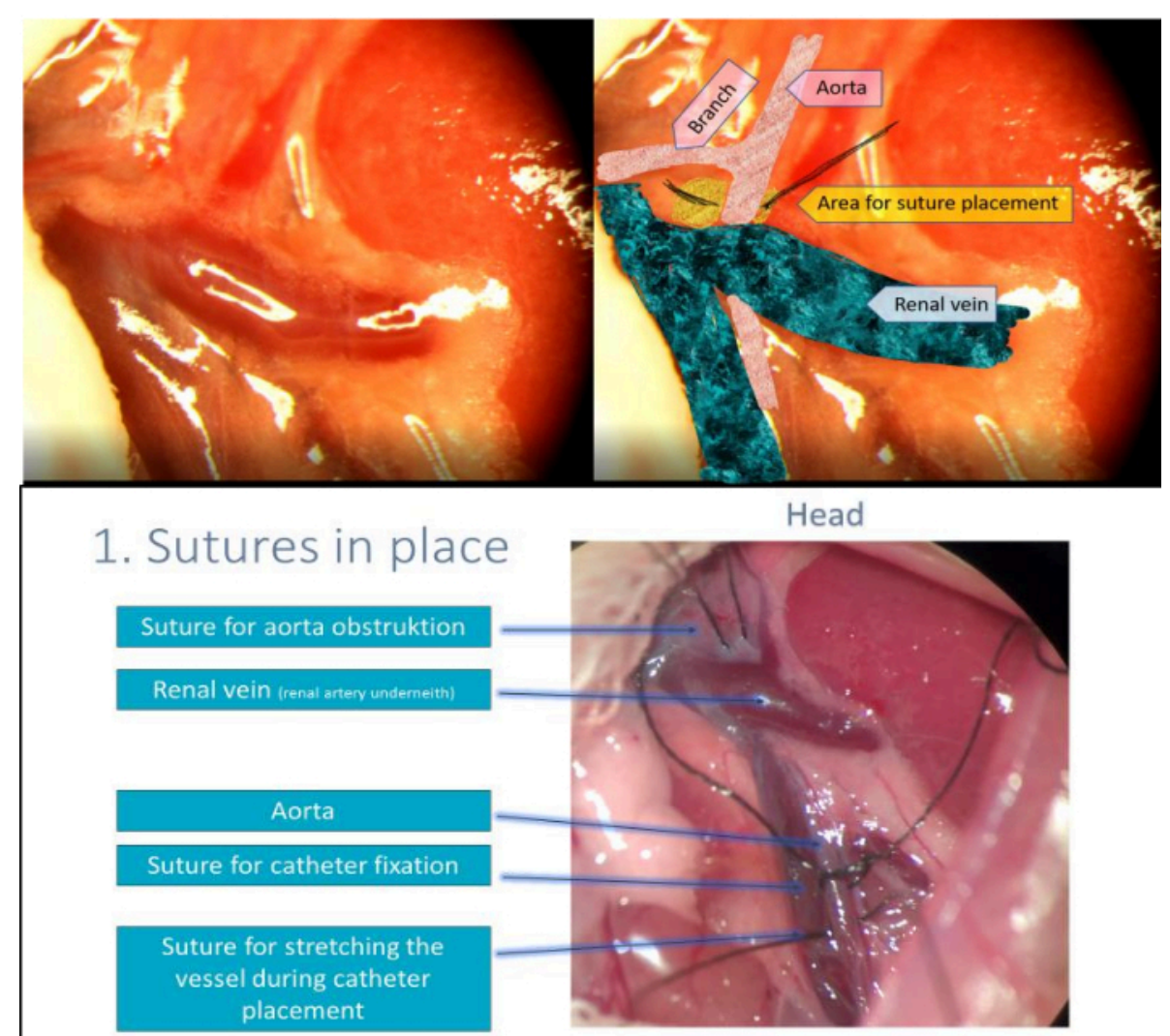

Tail

2. Catheter in place

Ischemic kidney (5-10 min)

Tight suture - aorta obstruktion

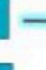

Dits

Head

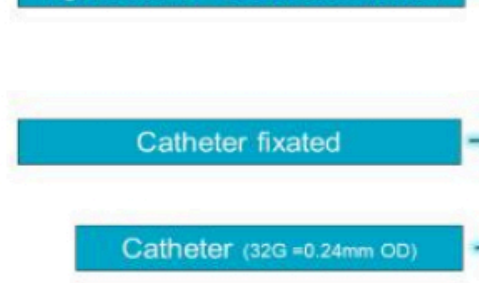

Catheter $(32 \mathrm{G}=0.24 \mathrm{~mm} \mathrm{OD})$
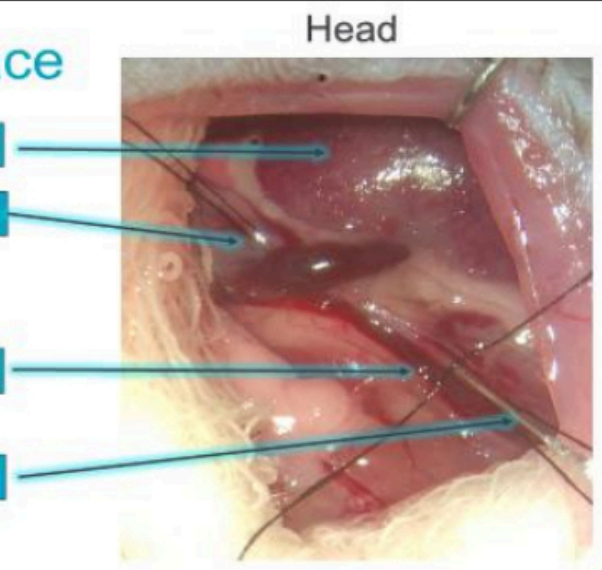

Tail

Figure 1: Placement of sutures and catheter. Please click here to view a larger version of this figure.

Top picture: Describing where to place the first ligature, cranially of the left kidney. Middle picture: Overview over the surgery area right before placement of catheter (2.11 in protocol). Bottom picture: Overview over the surgery area right before injection (2.10 in protocol) 
Pathological observations in response to intra-renal administration method

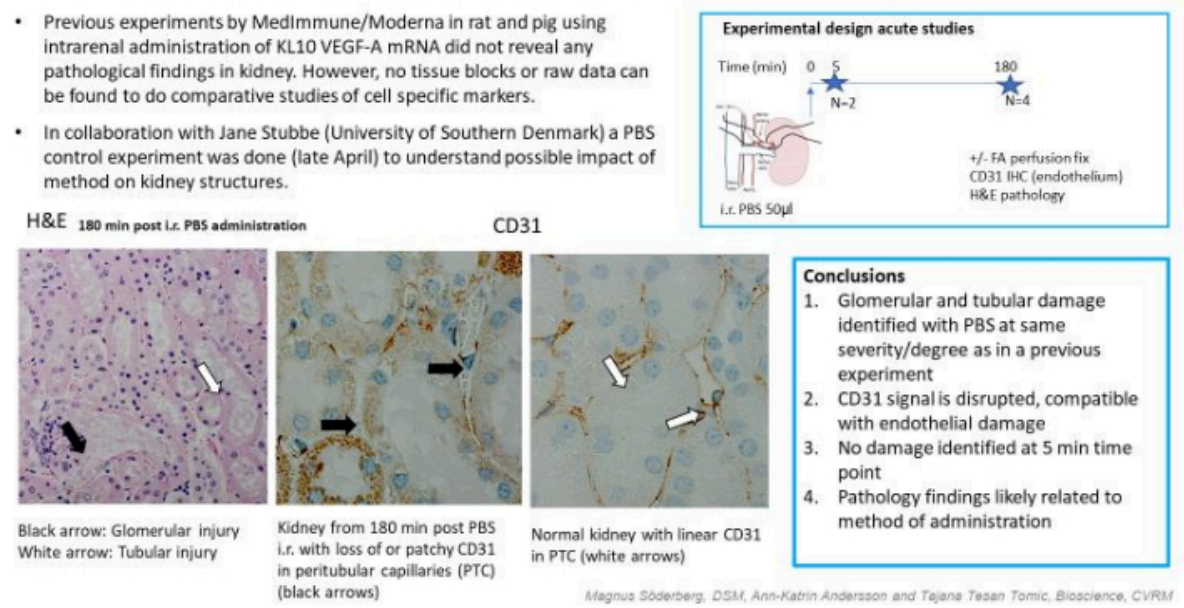

Figure 2: Injections directly into renal artery. Design of, and IHC results from, a study with injection of $\mathrm{NaCl}$ directly into the renal artery Please click here to view a larger version of this figure.

IHC results 7 days after aorta administration of $50 \mu \mathrm{IaCl}$

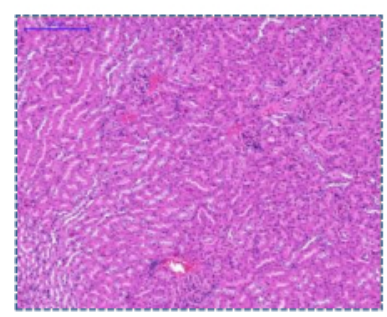

H\&E, normal

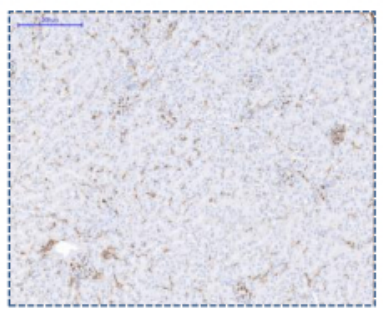

Desmin 0\%

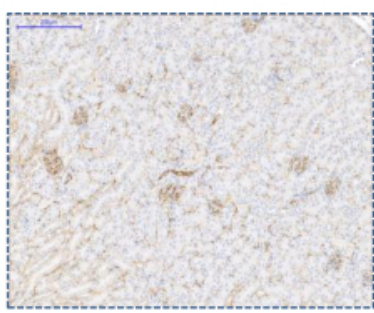

CD31, normal

Figure 3: IHC results at day 7 after aorta administration. IHC results from a study with injection of $\mathrm{NaCl}$ into the renal artery via abdominal aorta, 7 days after injection Please click here to view a larger version of this figure. 


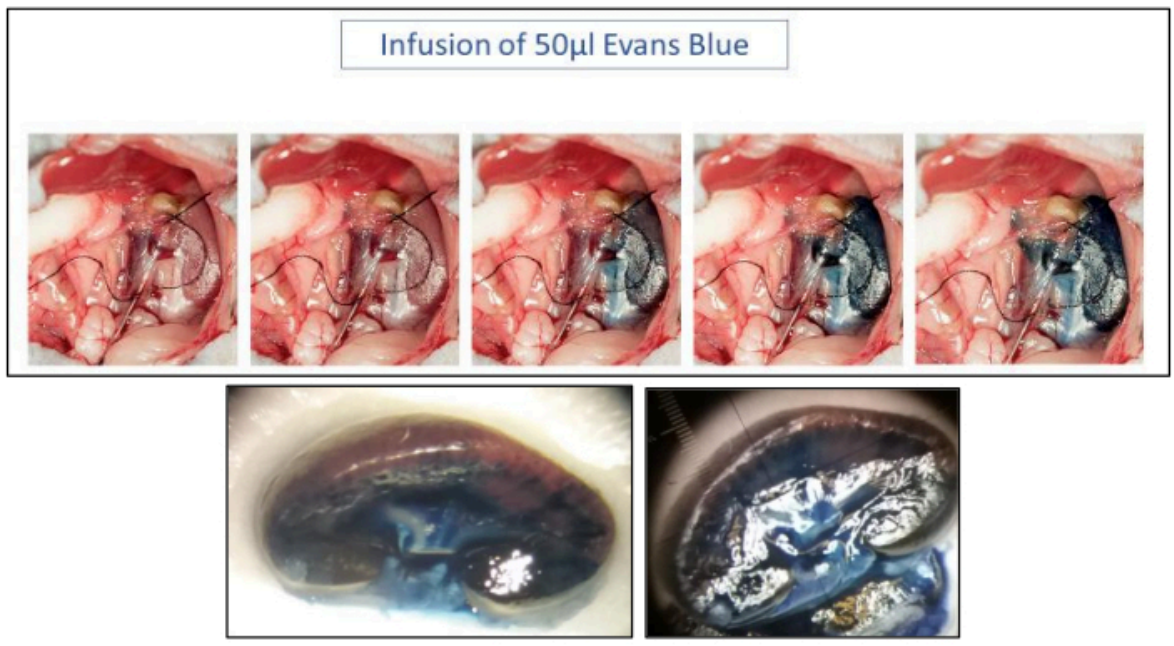

Figure 4: Injection of Evans blue dye. Top picture showing a serial view of an injection via the aorta of Evans blue dye. Bottom picture showing a cross-section of a kidney after injection via aorta of Evans blue dye. Please click here to view a larger version of this figure.
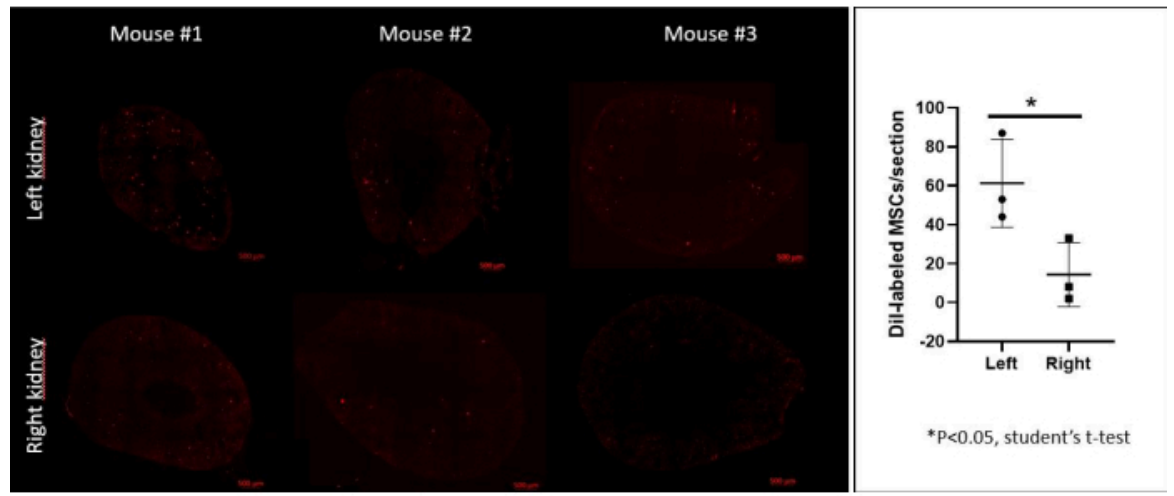

Figure 5: Abdominal arterial infusion significantly increased engraftment of mesenchymal stem cells in the left kidney compared to the right kidney. Human bone marrow derived mesenchymal stem cells were labeled with CM-Dil (red fluorescence) in suspension. One million labeled MSCs were infused through abdominal artery in three mice. Three hours after MSD infusion, mice were terminated. Left and right kidneys were harvested and embedded in OCT for cryosectioning. Please click here to view a larger version of this figure. 


\section{Discussion}

This method has been successful in delivering formulations to the kidney without causing renal damage. It can be used for delivery of any kind of formulations (e.g., small molecules, stem/progenitor cells, or microvesicles). The method can be applied in healthy animals or in renal disease models.

In Figure 2 and Figure 3, the histology is presented. Figure 2 shows histology 180 minutes after perfusion while Figure 3 shows history 7 days after infusion. The reason for this is that the experiments with renal artery was only 180 minutes long. To be sure we had no chronic damage with this new method, we purposely waited 7 days to evaluate them. The $n$-number is small but this is just an example.

Note that this newly developed method of aorta administration to target the kidney is an invasive method with relatively low throughput and is suitable for single dosing strategies only. Repeated dosing is not possible with this method.

With minor adjustments of the ligature placements this method could possibly be used to infuse both kidneys simultaniously ${ }^{5,7}$. With the increased risk of spillover through aortic branching we decided to keep the occluding ligature as close to the kidney as possible, leading the injection to the left kidney only. It can also be used in addition to other models or techniques like uninephrectomy or perhaps even simultaneous renal ischemia reperfusion injury (IRI). In our opinion, doing the injection only 24 hours after $|R|^{3}$ surgery is too harsh and will affect the animal's recovery substantially.

When using new strains of mice, one should always perform a pilot experiment on some animals from that strain to ensure that the method does not need to be adjusted in some way. For example, in some strains the vessel-branching from the aorta can be positioned a bit different. The position of the ligatures may change somewhat to ensure that the formulation ends up at the right location.

\section{Dehydration}

Opening the abdominal cavity poses a great risk for dehydration. It is therefore imperative to add fluids (around $37^{\circ} \mathrm{C}$ ) both during and after surgery to ensure good basis for recovery post operatively. We usually give $1 \mathrm{~mL} / \mathrm{mice}$ s.c. post-surgery.

\section{Ischemia}

Prolonged surgery time or if failing to reach full recirculation will result in ischemia. It is important to record the actual ischemia time and make sure that circulation to the kidney (and back legs) are restored. Constant training and refreshing of skills are, as always, important. Performing the procedure faster reduces the time the kidney is exposed to ischemia. Keeping the ischemia time (ligation of aorta) to around $5 \mathrm{~min}$ (10 min max) has been shown not to harm the kidney.

\section{Spillover}

Considering the risk of spillover to other organs when the injected formulation is re-entering the circulation through the renal vein, the injected volume should be kept to a minimum. We recommend a maximum volume of $50 \mu \mathrm{L}$. Dosage has also been found, by others, to be a critical factor for efficiency ${ }^{3}$.

Adverse effects (thrombosis and paralysis)

Initially when setting up the model we experienced nonconsistent problems with paralysis in the back legs. After relevant dose of anticoagulant (10 IU of heparin in tail vein) preoperatively, this adverse effect was significantly decreased. After consulting experts in the field, we also started to be even more careful in working with atraumatic surgery, meaning no pinching in any tissues and touching as 
little areas as possible. These actions taken together reduced the frequency of paralysis from around $50 \%$ to virtually none.

Initially during method development, we applied anticoagulant tissue on the renal artery to close the incision hole. This was not possible when doing injections into the aorta since the pressure is so much higher in the aorta. Anticoagulant also increases the risk of thrombosis if it enters the vessel lumen.

Make sure not to have adventitia exposed in the lumen of the aorta when suturing since adventitia is very thrombogenic. Also avoid any inverting wound edges or narrowing the aorta by suturing to thigh.

We now have an established method for delivering formulations directly to the kidney.

\section{Disclosures}

The author has nothing to disclose.

\section{Acknowledgments}

Thanks to René Remie at RRSSC for helping me finetune the technique and Instech labs for collaboration in producing a special designed catheter. A big thanks also to all colleagues at AstraZeneca R\&D for productive discussions with tips and tricks as well as Xerox for completing the video with sound.

\section{References}

1. Porvasnik, S.L., Mah, C., Polyak. S. Targeting Murine Small Bowel and Colon Through Selective Superior Mesenteric Artery Injection. Microsurgery. 30 (6), 487-93 (2010).

2. Rocca, C.J., Ur, S.N., Harrison, F., Cherqui, S. rAAV9 combined with renal vein injection is optimal for kidney- targeted gene delivery: conclusion of a comparative study. Gene Therapy. 21 (6), 618-628 (2014).

3. Cai, J. et al. Maximum efficacy of mesenchymal stem cells in rat model of renal ischemia-reperfusion injury: renal artery administration with optimal numbers. PLoS One. 9 (3), e92347 (2014).

4. Leda, A.R., Dygert, L., Bertrand, L., Toborek, M. Mouse Microsurgery Infusion Technique for Targeted Substance Delivery into the CNS via the Internal Carotid Artery. Journal of Visualized Experiments. .(119), e54804 (2017).

5. Monteiro Carvalho Mori da Cunha, M.G. et al. A Surgical Technique for Homogenous Renal Distribution of Substances in Rats. European Surgical Research. 51, 58-65 (2013).

6. De Jong, W.H. et al. Particle size-dependent organ distribution of gold nanoparticles after intravenous administration.Biomaterials. 29 (12), 1912-9 (2008).

7. Ullah, M. et al. A Novel Approach to Deliver Therapeutic Extracellular Vesicles Directly into the Mouse Kidney via Its Arterial Blood Supply. Cells. 9 (4), 937 (2020). 